\title{
HOLOMORPHIC CONTINUATION OF SMOOTH FUNCTIONS OVER LEVI-FLAT HYPERSURFACES $\left({ }^{1}\right)$
}

\author{
BY
}

ERIC BEDFORD

\begin{abstract}
Here we consider the singularities of a Levi-flat real hypersurface $S$ in $C^{n}$ that lie in an analytic variety of codimension 2 . It is shown that, from the geometric point of view, there are two kinds of singularities, and the type of singularity determines whether $S$ bounds a domain of holomorphy of type $A^{\infty}$.
\end{abstract}

1. Introduction. A real hypersurface $S$ in an open subset of $C^{n}$ is called a $C^{1}$ analytic hypersurface if locally $S$ is foliated by a $e^{1}$ family of varieties of codimension 1. That is, for each point $p \in S$, there is a neighborhood $N \subseteq C^{n}$ containing $p$ and a $e^{1}$ function $f(z, t), f: N \times R \rightarrow C$ which is holomorphic in $z$ and which has the properties:

(i) $S \cap N=\{z \in N: f(z, t)=0$ for some $t \in R\}$;

(ii) $d_{z} f(z, t), d_{t} f(z, t) \neq 0$ if $f(z, t)=0$.

An analytic hypersurface is easily seen to be pseudoconvex from both sides. Conversely, a theorem of E. Levi states that a $e^{2}$ hypersurface is an analytic hypersurface if and only if its Levi form vanishes (see Vladimirov [8, p. 164]).

In this paper we look at singularities of analytic hypersurfaces in $C^{n}$ that are varieties of codimension 2 , and we discuss the continuation of smooth (to the boundary) holomorphic functions over such singularities. Let us consider an analytic hypersurface $S$ in $\Omega=B(0,1) \backslash E$, where $E$ is a variety of codimension 2 in $B(0,1)=\left\{z \in C^{n}:|z|<1\right\}$.

As an additional assumption on $S$, we require that $S$ is foliated by varieties $V$ in $B(0,1) \backslash E$. Since $E$ is a removable singularity for a variety of dimension $n-1$, the closure of $V, \bar{V} \cap B(0,1)$ is again a variety. Our assumption is stated as follows:

(iii) for some $\varepsilon>0$ there is a $e^{1}$ function $f(z, t)$ which is analytic for $z \in B(0,1+\varepsilon)$ and periodic in $t$ (with period 1$)$ and such that

Received by the editors March 26, 1976.

AMS (MOS) subject classifications (1970). Primary 32D05, 32D15; Secondary 32C40.

(1) This research was supported in part by a Sloan Foundation grant to the Courant Institute of Mathematical Sciences, New York University, and Army Research Office Number DAHC04-74-G-0159. 


$$
B(0,1) \cap \bar{S}=\{|z|<1: f(z, t)=0 \text { for some } t\} .
$$

With condition (iii) it is clear that our emphasis is on the foliation of $S$ rather than on the vanishing of the Levi form, and for that reason we use the term analytic hypersurface rather than Levi-flat hypersurface. The notation

$$
V(t)=\{z \in B(0,1) \backslash E: f(z, t)=0\}
$$

and $\bar{V}(t)=\{z \in B(0,1): f(z, t)=0\}$ will be used from now on. Since $S$ is a $e^{1}$ surface we may assume without loss of generality that $V\left(t_{1}\right) \cap V\left(t_{2}\right)=\varnothing$ if $t_{1} \neq t_{2}$. The following result shows that there are two kinds of behavior at a singularity.

THEOREM A. Let $E$ be an irreducible variety of codimension 2 in $B(0,1) \subseteq C^{n}$, and let $S$ be an analytic hypersurface in $B(0,1) \backslash E$ satisfying (i), (ii) and (iii). Then if $z_{0} \in E$, either $z_{0} \in \bar{V}(t)$ for at most one $t$, or $E \subseteq \bar{V}(t)$ for all $t$.

In the first case $E$ will be said to be an inessential singularity of $S$; otherwise $E$ is essential. Note that although a singularity may be inessential, the variety $V\left(t_{0}\right)$ passing through $E$ may be singular, and $\bar{S} \cap B(0,1)$ may not even be a manifold. For instance, the analytic hypersurface $S=\left\{z \in C^{2}: 0<|z|\right.$ $\left\langle 1, z_{1} z_{2}=t\right.$ for some $\left.t \in(-1,1)\right\}$ fails to be a manifold at $(0,0)$ because

$$
S \cap\{|z|=\varepsilon\}=\left\{\left(r e^{i \theta}, \pm \sqrt{\varepsilon^{2}-r^{2}} e^{-i \theta}\right),|r| \leqslant \varepsilon, 0 \leqslant \theta \leqslant 2 \pi\right\}
$$

is a torus.

The main result of this paper is the following theorem which allows us to view an analytic hypersurface as the restriction of a larger foliation.

THEOREM B. Let $S$ be a $e^{2}$ analytic hypersurface in $B(0,1) \backslash E$ satisfying (i), (ii) and (iii). Then there is a $\mathrm{C}^{1}$ foliation $\{V(\theta+i \eta): 0 \leqslant \theta \leqslant 1,|\eta|<\delta\}$ of $a$ neighborhood of $S$ by varieties of codimension 1 which extends the foliation $\{V(\theta): 0 \leqslant \theta \leqslant i\}$ of $S$.

THEOREM $\mathrm{B}^{\prime}$. Let $S$ be an analytic hypersurface in $B(0,1) \backslash E$ satisfying (i), (ii), and (iii), and let the function $f(z, \theta)$ in (iii) be real analytic. Then there is a holomorphic foliation $\left\{V^{\prime}(\zeta):|\operatorname{Im} \zeta|<\delta, 0 \leqslant \operatorname{Re} \zeta \leqslant 1\right\}$ of a neighborhood of $S$ by varieties of codimension 1 with the property that $V^{\prime}(\theta)=V(\theta)$ if $0 \leqslant \theta \leqslant 1$.

It should be noted that the foliations in Theorems $B$ and $B^{\prime}$ are not the same in general. If $f(z, \theta)$ is real analytic, then the foliation in Theorem B will be real analytic but not always holomorphic. If the singular set $E$ is empty, then Theorem $\mathrm{B}^{\prime}$ is contained in Theorem 5.1 of Rea [10].

For an open set $D \subseteq C^{n}$, let $\theta(D)$ denote the holomorphic functions and $A^{j}(D)=O(D) \cap C^{j}(\bar{D})$ denote the holomorphic functions that have $j$ continuous derivatives up to the boundary. A domain of holomorphy $D \subseteq C^{n}$ 
will be called a domain of holomorphy of type $A^{j}$ if there exists a function $f \in A^{j}(D)$ that cannot be continued analytically to a larger domain. The following theorem tells when analytic hypersurfaces bound domains of holomorphy of type $A^{\infty}$.

THEOREM C. Let $S$ be a $\mathcal{C}^{2}$ analytic hypersurface in $B(0,1) \backslash E$, and let $\Omega^{ \pm}$be the connected components of $B(0,1) \backslash \bar{S}$. Then $E$ is an inessential singularity for $S$ if and only if $\Omega^{ \pm}$are of type $A^{\infty}$.

The main technical tool used to prove Theorem $\mathrm{C}$ is reminiscent of the sliding "disk" lemma; see Vladimirov [8, p. 150]. In the present context, however, the disk is not slid but rather wiggled, using the foliation of Theorem B.

Whether or not $E$ is an essential singularity, the sets $\Omega^{ \pm}$are easily seen to be domains of holomorphy. In fact more is true.

THEOREM D. If $S$ is $\mathcal{C}^{2}$ and the domains $\Omega^{ \pm}$are as in Theorem $\mathrm{C}$, then $\Omega^{ \pm}$are domains of holomorphy of type $A^{j}$ for all $j<\infty$.

As a special case of Theorems $\mathrm{C}$ and $\mathrm{D}$, we look at specific domains.

COROLlaRY 1. Let $\Omega^{\prime} \subseteq C^{n}$ be a domain of type $A^{\infty}$, let $f_{1}(z), \ldots, f_{j}(z)$ be in $A^{\infty}\left(\Omega^{\prime}\right)$, and suppose that

$$
\Omega=\left\{z \in \Omega^{\prime}:\left|f_{1}(z)\right|<\left|f_{2}(z)\right|<\cdots<\left|f_{j}(z)\right|\right\}
$$

where $f_{1}$ does not vanish identically on any component of $\Omega^{\prime}$. If the set

$$
T=\left\{z \in \Omega^{\prime}: f_{1}(z)=f_{2}(z)=0<\left|f_{3}(z)\right|<\cdots<\left|f_{j}(z)\right|\right\}
$$

has codimension 2, then every $f \in A^{\infty}(\Omega)$ may be continued to a neighborhood of $T$. On the other hand, if $m<\infty$ then $\Omega$ is a domain of holomorphy of type $A^{m}$.

It would be of interest to know what happens to Theorems C and D if assumption (iii) is dropped or if the set $E$ is allowed to be larger. Observe that the singularity $E$ is the smallest possible, for if two varieties $V_{1}, V_{2} \subseteq C^{n}$ of complex dimension $n-1$ intersect, then intersection $V_{1} \cap V_{2}$ must have dimension $\geqslant n-2$.

Let us outline the contents of the following sections. In \$2, the basic example is given for continuation over analytic hypersurfaces, with some more general examples of domains of holomorphy of type $A^{\infty}$. Theorem $\mathrm{C}$ is proved in $\$ 3$, and then the proof of Corollary 1 is given. In $\$ 4$, some results concerning the degree of a holomorphic mapping are given which lead both to a proof of Theorem A (easy) and a proof of Theorem B, which is completed 
in $\$ 5$. The variations for Theorem $B^{\prime}$ are outlined at the end of $\$ 5$. The proof of Theorem $\mathrm{D}$ is given in $\$ 6$.

2. Examples. The work that follows is motivated by the example given here. Set $\Omega^{+}=\left\{z \in C^{2},\left|z_{1}\right|^{p / q}<\left|z_{2}\right|<1\right\}$. The domain of holomorphy $\Omega^{+}$is bounded by the analytic hypersurface

$$
S=\left\{z \in C^{2}, 0<|z|<1,\left|z_{1}\right|^{p}=\left|z_{2}\right|^{q}\right\}=\bigcup_{t \in[0,1]} V(t)
$$

where $V(t)=\left\{0<|z|<1, z_{1}^{p}=e^{2 \pi i t} z_{2}^{q}\right\}$. Observe that in general the domain $\Omega^{+}(\alpha)=\left\{z \in C^{2}:\left|z_{1}\right|^{\alpha}<\left|z_{2}\right|<1\right\}$ is bounded by an analytic hypersurface. But if $\alpha$ is irrational, the leaves $V(\theta)=\left\{e^{i \theta} z_{1}^{\alpha}=z_{2}\right\}$ are dense in the set $\left\{\left|z_{1}\right|^{\alpha}=\left|z_{2}\right|\right\}$ and cannot be given as varieties in the set $\{0<|z|<1\}$. Thus, if $\alpha$ is irrational $\partial \Omega^{+}(\alpha)$ satisfies (i) and (ii) but not (iii).

It was shown by N. Sibony, [6, p. 976], that $\Omega^{+}$is not a domain of holomorphy of type $A^{\infty}$ by the following argument. Since $\Omega^{+}$is a circular domain, any $f \in O\left(\Omega^{+}\right)$has a Laurent expansion:

$$
f\left(z_{1}, z_{2}\right)=\sum_{n=-\infty}^{\infty} a_{n}\left(z_{1}\right) z_{2}^{n} .
$$

If $f \in A^{\infty}\left(\Omega^{+}\right)$, then $f\left(0, z_{2}\right)$ is holomorphic in the disk $\left\{\left|z_{2}\right|<1\right\}$. Thus $a_{n}(0)=0$ for $n=-1,-2, \ldots$ Similarly, $\left(\partial^{j} f / \partial z_{1}^{j}\right)\left(0, z_{2}\right)$ is holomorphic in $\left\{\left|z_{2}\right|<1\right\}$, and thus $\left(\partial^{j} / \partial z_{1}^{j}\right) a_{n}(0)=0$ for $n=-1,-2, \ldots$ Since $a_{n} \equiv 0$ for $n=-1,-2, \ldots$, it follows that $f$ is holomorphic at $(0,0)$.

Observe that $\Omega^{+}$is a domain of holomorphy of type $A^{j}$ for every $j<\infty$. For example $g\left(z_{1}, z_{2}\right)=z_{1}^{N+p+2} z_{2}^{-q} \in A^{N}\left(\Omega^{+}\right)$, but $g$ cannot be continued past $(0,0)$. By a theorem of Spallek [7], i.e., $e^{N}$, weakly holomorphic $=$ holomorphic, there is an integer $N$ with the property that if $\left.g\right|_{V(t)} \in e^{N}(\bar{V}(t))$ $\cap \theta(V(t))$, then $\left.g\right|_{V(t)} \in \theta(\bar{V}(t))$. But $g \notin A^{\infty}\left(\Omega^{+}\right)$as this example shows.

Other analytic hypersurfaces may be obtained in the following manner. Let $f_{1}$ and $f_{2}$ be analytic near the origin in $C^{n}$, and suppose that $E=\{z$ : $\left.f_{1}(z)=f_{2}(z)=0\right\}$ has codimension 2 . Then the surface $S=\left\{z \notin E:\left|f_{1}(z)\right|\right.$ $\left.=\left|f_{2}(z)\right|\right\}$ is an analytic hypersurface which is foliated by $V(t)=\{z \notin E$ : $\left.f_{1}(z)=e^{i t} f_{2}(z)\right\}$. If the varieties $V(t)$ are nonsingular, $S$ will be a real analytic hypersurface and will satisfy (i), (ii) and (iii). Since $E \subseteq \bar{V}(t)$, the singularity $E$ is essential.

If one asks in general what domains of holomorphy $\Omega \subseteq C^{n}$ are also domains of type $A^{j}$, an immediate sufficient condition is that there exist domains of holomorphy $U_{\alpha}$ such that $\bar{\Omega} \subset U_{\alpha}$ and $\cap_{\alpha} U_{\alpha}=\bar{\Omega}$, i.e., that $\Omega$ be its own nebenhull. A strongly pseudoconvex domain for example will have this property. But an example of Diederich and Fornaess [1] and [2] shows that a 
smooth domain of holomorphy may be type $A^{\infty}$ without having such a system of Stein neighborhoods.

If $\Omega$ is an open set in $C^{n}$, then $A^{j}(\Omega)$ is a Fréchet space. The argument in Lelong $[4$, p. 31$]$ may be modified slightly to prove the following result.

Proposition 1. Let $E$ be a dense subset of $\partial \Omega$ with the property that for each $x \in E$ there is an $f_{x} \in A^{j}(\Omega)$ which cannot be continued past $x$. Then $\Omega$ is a domain of holomorphy of type $A^{j}$.

Proposition 1 in combination with a theorem of Kohn [3] on solvability of the $\bar{\partial}$-operator for smooth forms yields a general class of domains of type $A^{\infty}$. Although the results in [3] are stated only for forms with coefficients in $C^{j}$, they remain valid for $C^{\infty}$, as was announced by Kohn.

Proposition 2. Suppose $\Omega \subseteq C^{n}$ is a domain of holomorphy with smooth boundary, and there exists a dense subset $\left\{p_{k}\right\} \subseteq \partial \Omega$ with the property that each $p_{k}$ has a neighborhood $U_{k}$ and $\Omega \cap U_{k}$ may be made strictly convex after $a$ holomorphic change of coordinates. Then $\Omega$ is of type $A^{\infty}$.

PROoF. If $p_{k}=0$ and $\left\{x_{1} \leqslant 0\right\}$ is a supporting half-space for $\Omega \cap U_{k}$, then consider the $(0,1)$-form $\mu=z_{1}^{-1} \bar{\partial} \chi$, where $\chi \in C_{0}^{\infty}\left(U_{k}\right)$ and $\chi=1$ is a neighborhood of 0 . Since $\Omega \cap U_{k}$ is strictly convex, $\mu$ is a smooth $\bar{\partial}$-closed $(0$, 1)-form on $\bar{\Omega}$, and thus there exists $h \in C^{\infty}(\bar{\Omega})$ such that $\bar{\partial} h=\mu$. It follows that $f(z)=\exp \left(z_{1}^{-1} \chi(z)-h(z)\right) \in A^{\infty}(\Omega)$ but $f$ cannot be continued analytically past 0 .

Certain analytic polyhedra are also of type $A^{\infty}$. Let $\Omega^{\prime}$ be a domain of type $A^{\infty}$ and let $f_{1}, \ldots, f_{N} \in A^{\infty}\left(\Omega^{\prime}\right)$ be given. Then the polyhedron $\Omega=\{z$ $\left.\in \Omega^{\prime}:\left|f_{j}(z)\right|<1, j=1,2, \ldots, N\right\}$ is of type $A^{\infty}$. For a point $p$ of the boundary

$$
\partial \Omega \subseteq \partial \Omega^{\prime} \cup \bigcup_{j=1}^{N}\left\{z \in \Omega^{\prime}:\left|f_{j}(z)\right|=1\right\},
$$

we must show that there exists a function $h \in A^{\infty}(\Omega)$ that cannot be continued past $p$. Since $\Omega^{\prime}$ is of type $A^{\infty}$, we need consider only $p \in \Omega^{\prime}$ such that $\left|f_{j}(p)\right|=1$. If $f_{j}$ cannot be continued past $p$, then we are finished. Otherwise, we may assume that $f_{j}(p)=1$, and we may take

$$
h(z)=\exp \left(f_{j}(z)-1\right)^{-1}
$$

In connection with Corollary 1 , note that $\Omega$ may also be defined as

$$
\Omega=\left\{z \in \Omega^{\prime}:\left|g_{N}(z)\right|<\left|g_{N-1}(z)\right|<\cdots<\left|g_{1}(z)\right|<1\right\}
$$

where $g_{k}(z)=\prod_{j=1}^{k} f_{j}(z)$. More generally, if $g_{1}, \ldots, g_{N} \in A^{\infty}\left(\Omega^{\prime}\right)$, then $\Omega$ 
will be of type $A^{\infty}$ if $g_{N}$ does not vanish identically on any component of $\Omega^{\prime}$ and if $g_{N-1}$ does not vanish on $\bar{\Omega}^{\prime}$.

3. Proof of Theorem C. It will be necessary to be able to push the leaves of an analytic hypersurface slightly so that they do not intersect the surface. If the singularity is inessential, this may be done very easily.

Leman 1. Suppose $S=\{z \in B(0,1) \backslash E: f(z, \theta)=0$ for some $\theta \in(-1,1)\}$ is an analytic hypersurface satisfying (i), (ii) and (iii) and that

$$
f(z, \theta)=f(z, 0)+\theta g(z)+R(z, \theta)
$$

where $f(z, 0), g(z)$ and $R(z, \theta)$ are analytic in $z$,

(a) $\sup _{|z|<1}|R(z, \theta)|=o(\theta)$,

(b) $\inf _{z \in V(0)}|g(z)|>0$.

Define, for $\eta \in R, W(\eta)=\{z \in B(0,1): f(z, 0)+i \eta g(z)=0\}$. Then there exists $\eta_{0}>0$ such that $W(\eta) \cap \bar{S}=\varnothing$ for $0<|\eta|<\eta_{0}$.

Proof. It suffices to show that there exists $\delta>0$ such that if $0<|\eta|<\delta$ and $|\theta|<\delta$, then $V(\theta) \cap W(\eta)=\varnothing$. By continuity, there exists $\delta>0$ such that $\inf _{z \in V(\theta)}|g(z)|>0$ for $|\theta|<\delta$.

If $z \in V(\theta) \cap W(\eta)$ then

$$
0=f(z, 0)+i \eta g(z), \quad 0=f(z, 0)+\theta g(z)+R(z, \theta) .
$$

This is equivalent to $z \in V(\theta)$ and $(i \eta / \theta-1) g(z)=R(z, \theta) / \theta$. Thus

$$
\left|\frac{R(z, \theta)}{\theta}\right| \geqslant\left(\frac{1}{2}\left|\frac{\eta}{\theta}\right|+1\right)|g(z)| \geqslant|g(z)|
$$

for some $z \in V(\theta)$. By (a), $\delta>0$ may be picked small enough that, if $|\theta|<\delta$,

$$
\inf _{|\theta|<\delta} \inf _{z \in V(\theta)}|g(z)|>\sup _{|z|<1}|\theta|^{-1}|R(z, \theta)|,
$$

and thus $V(\theta) \cap W(\eta)=\varnothing$.

Proof that if $E$ is inessential, then $\Omega^{+}$is a domain of holomorphy of type $A^{\infty}$. Let $f(z, \theta)$ be a defining function for $S$. By Proposition 1 it will suffice, for a dense set of $\theta$, to construct a function $h_{\theta}(z) \in A^{\infty}\left(\Omega^{+}\right)$which cannot be continued past $V(\theta)$.

Let us first claim that if $E$ is inessential, then except for finitely many values $\theta_{1}, \ldots, \theta_{m}$ of $\theta$ ( $m$ is the number of connected components of $E$ ),

$$
\left\{d_{\theta} f(z, \theta)=0\right\} \cap \nabla(\theta)=\varnothing .
$$

Otherwise, by condition (ii), $\left\{d_{\theta} f(z, \theta)=0\right\} \cap \nabla(\theta)=E^{\prime}$, where $E^{\prime}$ is a union of connected components of $E$. But if $E$ is inessential, the intersection 
can equal a fixed $E^{\prime}$ for at most one value of $\theta$. This establishes the claim.

Since the surface $S$ is defined on $B(0,1+\varepsilon)$, we may have $d_{\theta} f(z, \theta) \neq 0$ on the set $\{z \in B(0,1+\varepsilon): f(z, \theta)=0\}$, from which we conclude that

$$
\inf _{z \in V(\theta)}\left|d_{\theta} f(z, \theta)\right|=\mu(\theta)>0
$$

for $\theta \neq \theta_{1}, \ldots, \theta_{m}$. By Lemma 1 , there exists $\delta(\theta)>0$ such that the variety $W(\theta, \eta)=\{z \in B(0,1): f(z, \theta)+i \eta(d / d \theta) f(z, \theta)=0\}$ does not intersect $S$ if $0<|\eta|<\delta(\theta)$. Furthermore, changing the sign of $\eta$ if necessary, we conclude that $W(\theta, \eta) \subseteq{ }^{c} \Omega^{+}$.

Let $\phi(\zeta)$ be an analytic function in $C \backslash\left[\delta(\theta)^{-1}, \infty\right)$ with the property that $\phi(\zeta)$ dies faster than any power of $|\zeta|$ as $\zeta \rightarrow \infty$, e.g.,

$$
\phi(\zeta)=\exp \left(-\left(\zeta-\delta(\theta)^{-1}\right)^{1 / 2}\right)
$$

It follows that since $W(\theta, \eta) \cap S=\varnothing$ for $\eta \in(0, \delta(\theta))$

$$
h(z)=\phi\left(i \frac{d}{d \theta} f(z, \theta)(f(z, \theta))^{-1}\right) \in A^{\infty}\left(\Omega^{+}\right) .
$$

Since $\phi$ vanishes rapidly at $\infty, h(z)$ vanishes to infinite order at $V(\theta)$ and therefore cannot be continued holomorphically past it.

Proof that elements of $A^{\infty}$ can be continued through an essential singularity. Here we show how this half of Theorem $\mathrm{C}$ may be deduced from Theorem B. Given $f \in A^{\infty}\left(\Omega^{+}\right)$, we must find an analytic continuation of $f$ to a neighborhood of the origin. By Theorem B, there exists a foliation $V(\zeta)$ in a neighborhood of $S$. Pick a leaf $V(\zeta)$ contained in $\Omega^{+}$. By a suitable choice of coordinates, $0 \in E$,

$$
V^{+}=B(0, \varepsilon) \cap \bar{V}(\zeta) \cap\left\{z_{3}=\cdots=z_{n}=0\right\}
$$

is a 1-dimensional variety, and $V^{+} \cap E=0$. There exists $\delta>0$ such that $\operatorname{dist}\left(\partial V^{+}, \partial \Omega^{+}\right) \geqslant \delta$. The Cauchy estimate gives

$$
\left|D_{z}^{\alpha} f(z)\right| \leqslant C \alpha ! \delta^{-|\alpha|}
$$

for all $z \in \partial V^{+}$, since $f$ is analytic in a ball of radius $\delta$ about each $z$. Furthermore, $D_{z}^{\alpha} f$ is holomorphic on the local variety $V^{+} \backslash 0$ and continuous on $V^{+}$. Thus $D_{z}^{\alpha} f$ is weakly holomorphic on $\bar{V}^{+}$and satisfies the maximum principle, whence (3.1) holds for all $z \in V^{+}$. In particular if $z_{0} \in V^{+}$and $0<\left|z_{0}\right|<\delta$, then the power series $f(z)=\sum_{\alpha>0} C_{\alpha}\left(z-z_{0}\right)^{\alpha}$ converges in $B\left(z_{0}, \delta\right)$, which contains the origin.

Let us remark that in the proof of the second part of Theorem $\mathbf{C}$ the essential point was the existence of local varieties $V^{ \pm}$such that $V^{ \pm} \subset \Omega^{ \pm}$and $\bar{V} \cap \bar{S}=0$. In the proof of Corollary $1, V^{+}$will be found without the aid of 
Theorem B. In many specific examples one of the varieties, say $V^{+}$, may be taken to be a disk. If $\alpha \in C P^{n-1} \backslash C(S)$ is a line at positive distance from $C(S)$ (cf. Lemma 6), then there exists $\varepsilon>0$ such that $D(\varepsilon)=\{\zeta \alpha: \zeta \in C$, $|\zeta|<\varepsilon\}$ satisfies $D(\varepsilon) \cap \bar{S}=0$. Thus $D(\varepsilon) \backslash 0$ lies in either $\Omega^{+}$or $\Omega^{-}$, say $\Omega^{+}$. On the other hand it is often not as easy to find, by inspection, a variety $V^{-}$.

The following example illustrates this point. Let $S \subseteq C^{2}$ be the analytic hypersurface swept out by the varieties

$$
\begin{aligned}
V(\theta)=\{z & \in B(0,1) \backslash 0: \\
& \left.=z_{1}+e^{4 \pi i \theta} 2^{-k} z_{2}^{k}+e^{2 \pi i \theta} 2^{-k-1}\left(z_{1}^{k+1}+z_{2}^{k+1}\right)\right\} .
\end{aligned}
$$

Let $\Omega^{+}$be the component of $B(0,1) \backslash \bar{S}$ such that $\left\{z_{2}=0\right\} \cap \Omega^{+} \neq \varnothing$. Let $W$ be the variety of any polynomial of degree $\leqslant k$, and set $W(\varepsilon)=(B(0, \varepsilon) \backslash 0)$ $\cap W$. If $W(\varepsilon) \cap \bar{S}=\varnothing$, then $W(\varepsilon) \subseteq \Omega^{+}$.

Proof of CoRollary 1. We show here that if $f \in A^{\infty}(\Omega)$, then $f$ may be continued to a neighborhood of $T$. This is an instance where the variety $V^{+}$is found by inspection. For $z_{0} \in T$, there exists $\varepsilon>0$ such that if $z \in B\left(z_{0}, \varepsilon\right)$, then

$$
0<\frac{1}{2}\left|f_{3}\left(z_{0}\right)\right|<\left|f_{3}(z)\right|<\cdots<\left|f_{j}(z)\right| .
$$

Choose coordinates $z=\left(z_{1}, \ldots, z_{n}\right)$ such that $z_{0}=0$ and that the local variety

$$
V_{0}(\varepsilon)=\left\{z \in B(0, \varepsilon): f_{1}(z)=\frac{1}{2} f_{2}(z)=z_{3}=\cdots=z_{n}=0\right\}
$$

has dimension 0 ; this is possible since $\operatorname{codim} T=2$. For $\varepsilon>0$ sufficiently small $V_{0}(\varepsilon)=0$. Thus the 1-dimensional local variety

$$
V(\varepsilon)=\left\{z \in B(0, \varepsilon): f_{1}(z)=\frac{1}{2} f_{2}(z)=z_{3}=\cdots=z_{n}\right\}
$$

has the property that if $z \neq 0, z \in V(\varepsilon)$ then $0<\left|f_{1}(z)\right|<\cdots<\left|f_{k}(z)\right|$. Now let $\varepsilon>0$ be chosen small enough that $\left|f_{2}(z)\right|<\frac{1}{2}\left|f_{3}(0)\right|$ if $z \in B(0, \varepsilon)$. It follows by (3.2) that $V(\varepsilon) \backslash 0 \subset \Omega$, and 0 is the closure of $V(\varepsilon) \backslash 0$, and so the second part of the proof of Theorem $C$ applies to the variety $V^{+}=V(\varepsilon) \backslash 0$. Thus $f$ may be continued to a neighborhood of $z_{0}=0$.

To see that $\Omega$ is a domain of type $A^{m}$, note that

$$
\Omega=\bigcap_{k=1}^{j-1}\left\{z \in \Omega^{\prime}:\left|f_{k}(z)\right|<\left|f_{k+1}(z)\right|\right\},
$$

and that $\partial \Omega \subseteq \cup_{k=1}^{j-1}\left\{\left|f_{k}(z)\right|=\left|f_{k+1}(z)\right|\right\} \cup \partial \Omega^{\prime}$. By Theorem D, there exists $f \in A^{m}(\Omega)$ that cannot be continued over the set $\left\{z \in \Omega:\left|f_{k}(z)\right|=\left|f_{k+1}(z)\right|\right\}$, and thus $\Omega$ is of type $A^{m}$. 
4. The degree of an analytic mapping. Simple results using the degree of a holomorphic mapping will facilitate the proofs of Theorems A and B. Let a differentiable mapping $F$ of an open subset $W$ of $C^{n}, F: W \rightarrow C^{n}$, be given. For a relatively compact open subset $\Omega \subset W$ and a point $b \notin f(\partial \Omega)$, $\operatorname{deg}(f, \Omega, b)$ is defined. For some applications of degree theory to holomorphic maps, see Rabinowitz [5]. The basic facts we shall use are that whenever $\operatorname{deg}(f, \Omega, b)$ is defined:

$$
\begin{gathered}
\operatorname{deg}(f, \Omega, b) \geqslant 0, \\
\operatorname{deg}(f, \Omega, b)>0 \text { if and only if } b \in f(\Omega), \\
\operatorname{deg}\left(f_{0}, \Omega, b\right)=\operatorname{deg}\left(f_{1}, \Omega, b\right),
\end{gathered}
$$

if there exists a homotopy $F:[0,1] \times W \rightarrow C^{n}$ from $f_{0}$ to $f_{1}$ and $\operatorname{deg}(F(t), \Omega, b)$ is defined for $t \in[0,1]$.

The following elementary lemma will show that nonempty intersections are "generic" for a continuous 1-parameter family of varieties, even if the varieties are highly tangent at their intersection.

Lemma 2. Suppose functions $f_{k}(z, \theta): \overline{B(0,1)} \times(-1,1) \rightarrow C$ are given, $k=1$, $2, \ldots, n$, which are analytic in $z$ and continuous in $\theta$ and which define varieties $V_{k}(\theta)=\left\{z \in C^{n}:|z|<1, f_{k}(z, \theta)=0\right\}$. If $\operatorname{codim}\left(V_{1}(0) \cap \cdots \cap V_{j}(0)\right)=j$, then there exists $\delta_{0}>0$ such that

$$
\operatorname{codim}\left(V_{1}(\theta) \cap \cdots \cap V_{j}(\theta)\right)=j
$$

for $|\theta| \leqslant \delta_{0}$, and in particular $V_{1}(\theta) \cap \cdots \cap V_{j}(\theta) \neq \varnothing$. Furthermore, the intersection moves continuously in the following sense: If $\bigcap_{k=1}^{n} V_{k}(0)=0$ then given $\varepsilon>0$, there is a $\theta_{0}>0$ such that

$$
\varnothing \neq \bigcap_{k=1}^{n} V_{k}(\theta) \subseteq B(0, \varepsilon)
$$

for $|\theta| \leqslant \theta_{0}$.

Proof. If $\operatorname{codim}\left(V_{1}(0) \cap \cdots \cap V_{j}(0)\right)=j$, then after a linear change of coordinates

$$
0=\operatorname{dim}\left(V_{1}(0) \cap \cdots \cap V_{j}(0) \cap\left\{z_{j+1}=\cdots=z_{n}=0\right\}\right) .
$$

Without loss of generality, there exists $\eta>0$ such that

$$
0=V_{1}(0) \cap \cdots \cap V_{j}(0) \cap\left\{z_{j+1}=\cdots=z_{n}=0\right\} \cap B(0, \eta) .
$$

If $G(z, \theta)=\left(f_{1}(z, \theta), \ldots, f_{j}(z, \theta), z_{j+1}, \ldots, z_{n}\right)$, then $|G(z, \theta)|>0$ for $|z|=\eta$ 
and so $\operatorname{deg}(G(z, 0), B(0, \eta), 0)$ is defined and positive. Thus if $|\theta|<\delta_{0}$, and $\delta_{0}$ is sufficiently small that

$$
\inf _{|\theta|<\delta_{0}} \inf _{|z|=\eta}|G(z, \theta)|>0,
$$

then $0<\operatorname{deg}(G(z, \theta), B(0, \eta), 0)=\operatorname{deg}(G(z, 0), B(0, \eta), 0)$. From this it follows that $V_{1}(\theta) \cap \cdots \cap V_{j}(\theta)$ is nonempty and thus has codimension $\leqslant j$. If it has codimension $<j$, then two varieties $V_{k}(\theta)$ and $V_{m}(\theta)$ must have a component in common. But for $\delta_{0}$ small, this cannot happen, as $V_{k}(0)$ and $V_{m}(0)$ can have no common component.

Observe that the continuity result follows by setting $\eta=\varepsilon$ and selecting $\delta_{0}$ such that the inequality above holds.

Proof of Theorem A. It must be shown that if there are real numbers $t_{1} \neq t_{2}$ such that $\bar{V}\left(t_{1}\right) \cap \bar{V}\left(t_{2}\right) \neq \varnothing$, then $E \subseteq V(t)$ for all $t$. Consider the set $\Lambda=\{t \in[0,1]: \bar{V}(t) \supseteq E\}$. By assumption, $V\left(t_{1}\right) \cap V\left(t_{2}\right)=\varnothing$ and $E$ is irreducible, so $\bar{V}\left(t_{1}\right) \cap \bar{V}\left(t_{2}\right) \supseteq E$ and $t_{1}, t_{2} \in \Lambda$. Clearly $\Lambda$ is closed since $f$ is continuous.

For $t_{0} \in \Lambda, t_{0} \neq t_{1}$, it follows that $\operatorname{codim}\left(\nabla\left(t_{0}\right) \cap \nabla\left(t_{1}\right)\right)=2$. By Lemma 2 , then, there exists $\delta>0$ such that $\operatorname{codim}\left(\nabla(t), \nabla\left(t_{1}\right)\right)=2$ if $\left|t-t_{0}\right|<\delta$. Thus $\left(t_{0}-\delta, t_{0}+\delta\right) \subseteq \Lambda$, and is open. We conclude that $\Lambda=[0,1]$, which establishes Theorem $A$.

Now we shall obtain some lower bounds on the degree of a holomorphic mapping in terms of its order of vanishing at the origin. In particular, this will allow us to compute $\operatorname{deg}(F, B(0, \eta), 0)$ for $\eta>0$ arbitrarily small. The point of this is that if $\operatorname{deg}(F, B(0,1), 0)=\operatorname{deg}(F, B(0, \eta), 0)$ for all $\eta>0$, then $F^{-1}(0)$ $=0$.

If $f(z)$ is an analytic function defined in a neighborhood of 0 in $C^{n}$, then $f(z)=\sum_{j=0}^{\infty} f_{j}(z)$ where $f_{j}(z)$ is a homogeneous polynomial of degree $j$ in $z=\left(z_{1}, \ldots, z_{n}\right)$. The order of $f$ at 0 is $\operatorname{Ord} f=\min \left\{j: f_{j}(z) \not \equiv 0\right\}$.

The following result is easily derived from the Weierstrass preparation theorem.

LEMMA 3. Let $F=\left(f_{1}, \ldots, f_{n}\right)$ be a holomorphic mapping, $F: \overline{B(0,1)} \rightarrow C^{n}$, where $0 \notin F(\partial B(0,1))$. Then

$$
\operatorname{deg}(F, B(0, \eta), 0) \geqslant \prod_{j=1}^{n} \operatorname{Ord} f_{j}
$$

for all $\eta>0$ for which the degree is defined.

Using properties (4.1) and (4.2) of holomorphic mappings, one may obtain a sufficient condition for $F^{-1}(0)=0$.

COROLlary 2. $F=\overline{B(0,1)} \rightarrow C^{n}$ is a holomorphic mapping, 


$$
0 \notin F(\partial B(0,1)) \text { and } \operatorname{deg}(F, B(0,1), 0)=\prod_{j=1}^{n} \operatorname{Ord} f_{j} \text {, }
$$

then $F^{-1}(0)=0$.

The inequality of Lemma 3 is not very useful for mappings like $F(z)$ $=\left(z_{1}+z_{2}^{2}, z_{1}+z_{2}^{4}\right)$ where Ord $F=1$ and $\operatorname{deg}(F, B(0,1), 0)=2$. We shall compute the degree more accurately for mappings in $C^{2}$. The next lemma computes the degree at 0 for mappings $F=(f, g)$ where $d g \neq 0$ on $\{g=0\}$. The case for more general $g$ can be handled by counting multiplicities and then summing over the irreducible components of $\{g=0\}$.

LEMMA 4. Let $F=(f, g): \overline{B(0,1)} \rightarrow C^{2}$ be a holomorphic mapping with $0 \notin F(\partial B(0,1))$ and $F(0)=0$. Suppose $d g \neq 0$ on $\{g=0\}$, and let $V_{1}, \ldots, V_{k}$ be the irreducible components of $\{g=0\}$ at 0 . If $\tau \rightarrow\left(\phi_{j}(\tau), \psi_{j}(\tau)\right), j=1,2$, $\ldots, k$, is a normalization of $V_{j}$ in some neighborhood of 0 , then

$$
\lim _{\eta \rightarrow 0} \operatorname{deg}(F, B(0, \eta), 0)=\sum_{j=1}^{k} \operatorname{Ord} f\left(\phi_{j}(\tau), \psi_{j}(\tau)\right) .
$$

Proof. Without loss of generality, we may take a point $(c, 0)$ to be a regular point of $F$. For this, we must show that $J_{F}$ is nonsingular on $F^{-1}(c, 0)$ $\subseteq\{z: g(z)<0\}$. But $\operatorname{det} J_{F} d z_{1} \wedge d z_{2}=d f \wedge d g$, and so, replacing $f$ by $\left(1+\varepsilon z_{1}\right) f$ if necessary, we may obtain $\operatorname{dim}(\{g=0\} \cap\{d f \wedge d g=0\})=0$. Observe that this substitution, for small $\varepsilon$, changes neither degree nor order. Thus, for $\eta>0$ sufficiently small, $d f \wedge d g \neq 0$ on $(B(0, \eta) \backslash 0) \cap\{g=0\}$.

It follows that $\operatorname{deg}(F, B(0, \eta), 0)$ is equal to the number of points where $\{g(z)=0\}$ and $\{f(z)=c\}$. It is easily seen that $\operatorname{Ord} f\left(\phi_{j}(\tau), \psi_{j}(\tau)\right)$ is the number of roots, near zero, of the equation $f\left(\phi_{j}(\tau), \psi_{j}(\tau)\right)=c$ and is thus the number of points in $V_{j} \cap\{f(z)=c\}$. Summing over $j$, we obtain the desired result.

Let us now extend the function $f(z, \theta)$ of (iii) from $I=[0,1]$ to the strip $\{\zeta \in C: \operatorname{Re} \zeta \in[0,1]\}$ by setting $\zeta=\theta+i \eta$ and

$$
f(z, \zeta)=f(z, \theta)+i \eta \frac{d}{d \theta} f(z, \theta)
$$

Observe that the Cauchy-Riemann operator $\partial / \partial \bar{\zeta}=\frac{1}{2}(\partial / \partial \theta+i \partial / \partial \eta)$ annihilates $f$ on the set $\{\operatorname{Im} \zeta=0\}$. As before we set

$$
\begin{aligned}
& V(\zeta)=\{z \in B(0,1) \backslash E: f(z, \zeta)=0\}, \\
& \bar{V}(\zeta)=\{z \in B(0,1): f(z, \zeta)=0\} .
\end{aligned}
$$

We restrict ourselves in the following lemma to the case $n=2$, and $E$ will be taken to be a finite set. Define $F\left(\zeta_{1}, \zeta_{2}\right): \overline{B(0,1)} \rightarrow C^{2}$ by $F\left(z, \zeta_{1}, \zeta_{2}\right)$ 
$=\left(f\left(z, \zeta_{1}\right), f\left(z, \zeta_{2}\right)\right)$, and

$$
D\left(\zeta_{1}, \zeta_{2}\right)=\lim _{\varepsilon \rightarrow 0} \operatorname{deg}\left(F\left(\zeta_{1}, \zeta_{2}\right), B(0, \varepsilon), 0\right)
$$

if the degree is defined.

Lemma 5. Let $F\left(\zeta_{1}, \zeta_{2}\right), D\left(\zeta_{1}, \zeta_{2}\right)$ be defined as above, and suppose that $0 \notin F\left(\zeta_{1}, \zeta_{2}\right)(\partial B(0,1))$ for $\zeta_{1} \neq \zeta_{2}$ and $\zeta_{1}, \zeta_{2}$ in $I(\delta)=\{\zeta \in C:|\operatorname{Im} \zeta|<\delta$, $\operatorname{Re} \zeta \in I\}$. Then the limit defining $D\left(\zeta_{1}, \zeta_{2}\right)$ exists. If $\zeta_{2} \in I(\delta)$ has the property that $D\left(\theta_{1}, \zeta_{2}\right)$ is constant for all $\theta_{1} \in I, \theta_{1} \neq \zeta_{2}$, then there exists $\delta\left(\zeta_{2}\right)$ such that $D\left(\zeta, \zeta_{2}\right)$ is constant for $\zeta \in I\left(\delta\left(\zeta_{2}\right)\right), \zeta_{1} \neq \zeta_{2}$.

Proof. Since $0 \notin F\left(\zeta_{1}, \zeta_{2}\right)(\partial B(0,1))$, it follows that $\operatorname{dim} F\left(\zeta_{1}, \zeta_{2}\right)^{-1}(0)=0$ for $\zeta_{1}, \zeta_{2} \in I_{\delta}$. Thus $F\left(\zeta_{1}, \zeta_{2}\right)^{-1}(0)$ is finite and there exists $\varepsilon_{0}=\varepsilon_{0}\left(\zeta_{1}, \zeta_{2}\right)$ such that $B(0, \varepsilon) \cap F\left(\zeta_{1}, \zeta_{2}\right)^{-1}(0)=0$ for $\varepsilon<\varepsilon_{0}$. Thus $\operatorname{deg}\left(F\left(\zeta_{1}, \zeta_{2}\right), B(0, \varepsilon), 0\right)$ is constant for $\varepsilon<\varepsilon_{0}$ and $D\left(\zeta_{1}, \zeta_{2}\right)$ exists.

Let $V_{1}, \ldots, V_{k}$ be the irreducible components of $\left\{z: f\left(z, \zeta_{2}\right)=0\right\}$ at the origin. Then for $j=1,2, \ldots, k, V_{j}$ may be normalized by the map $\tau$ $\rightarrow\left(\phi_{j}(\tau), \psi_{j}(\tau)\right)$ in some neighborhood of the origin. Set

$$
f_{j}(\tau, \zeta)=f\left(\phi_{j}(\tau), \psi_{j}(\tau), \zeta\right)
$$

and write $f_{j}(\tau, \zeta)=\sum_{q=0}^{\infty} \tau^{q} f_{j}^{q}(\zeta)$.

Let us first consider the case $\theta_{1} \neq \zeta_{2}$, and show that there exists $\delta\left(\theta_{1}\right)$ such that $D\left(\zeta, \zeta_{2}\right)$ is constant for $\left|\zeta-\theta_{1}\right|<\delta\left(\theta_{1}\right)$. Observe that for $\zeta$ near $\theta_{1}$, $\operatorname{Ord} f_{j}(\tau, \zeta) \leqslant \operatorname{Ord} f_{j}\left(\tau, \theta_{1}\right)$, i.e., $\operatorname{Ord} f_{j}(\tau, \zeta)$ is upper semicontinuous. Since $D\left(\theta, \zeta_{2}\right)=\sum_{j=1}^{k} \operatorname{Ord} f_{j}(\tau, \theta)$ is constant for $\theta \neq \zeta_{2}$, it follows that $\operatorname{Ord} f_{j}(\tau, \theta)$ is constant in $\theta$. Since

$$
f_{j}(\tau, \zeta)=f_{j}\left(\tau, \theta_{1}\right)+\left(\zeta-\theta_{1}\right)(d / d \theta) f_{j}\left(\tau, \theta_{1}\right)+R\left(\tau, \operatorname{Re} \zeta, \theta_{1}\right)
$$

with $\left|R\left(\tau, \operatorname{Re} \zeta, \theta_{1}\right)\right|=o\left(\operatorname{Re}\left(\zeta-\theta_{1}\right)\right)$, it follows that

$$
\operatorname{Ord} f_{j}\left(\tau, \theta_{1}\right) \leqslant \operatorname{Ord} \frac{d}{d \theta} f_{j}\left(\tau, \theta_{1}\right) .
$$

Similarly $\operatorname{Ord} R(\tau, \operatorname{Re} \zeta, \theta) \geqslant \operatorname{Ord} f_{j}\left(\tau, \theta_{1}\right)$. Thus we may take $\delta\left(\theta_{1}\right)<\left|\operatorname{Im} \zeta_{2}\right|$ if $\operatorname{Im} \zeta_{2} \neq 0$ and $\delta\left(\theta_{1}\right)<\left|\theta_{1}-\zeta_{2}\right|$ if $\operatorname{Im} \zeta_{2}=0$.

For the case $\theta_{1}=\zeta_{2}$, observe that $f_{j}\left(\tau, \theta_{1}\right)=0$ for all $\tau$, and, by (ii), $(d / d \theta) f_{j}\left(\tau, \theta_{1}\right) \neq 0$. Thus for $\zeta$ near $\theta_{1}$, we write

$$
f_{j}(\tau, \zeta)=\left(\zeta-\theta_{1}\right)(d / d \theta) f_{j}\left(\tau, \theta_{1}\right)+R_{j}\left(\tau, \theta_{1}, \theta\right) .
$$

If $\operatorname{Im} \zeta=0$ and $\zeta \neq \theta_{1}$, then $\operatorname{Ord} f(\tau, \zeta)=q$ is constant. Thus

$$
q=\operatorname{Ord}(d / d \theta) f_{j}\left(\tau, \theta_{1}\right) \leqslant \operatorname{Ord} R_{j}\left(\tau, \theta_{1}, \theta\right) .
$$


Let $\delta^{\prime}>0$ be chosen so that

$$
\left|\theta-\theta_{1}\right|\left|(d / d \theta) f_{j}^{q}\left(\theta_{1}\right)\right|>\left|R_{j}^{q}\left(\theta_{1}, \theta\right)\right|
$$

if $\left|\theta-\theta_{1}\right| \leqslant \delta^{\prime}$. Thus if $\left|\xi-\theta_{1}\right|<\delta^{\prime}$ it follows that

$$
\begin{aligned}
\left|f_{j}^{q}(\zeta)\right| & \geqslant|\theta+i \eta|\left|(d / d \theta) f_{j}^{q}\left(\theta_{1}\right)\right|-\left|R_{j}^{q}\left(\theta_{1}, \theta\right)\right| \\
& \geqslant \eta\left|(d / d \theta) f_{j}^{q}\left(\theta_{1}\right)\right|>0,
\end{aligned}
$$

whence $\operatorname{Ord} f_{j}(\tau, \zeta)=q$.

By the compactness of $I$ it now follows that $\delta\left(\zeta_{2}\right)>0$ may be chosen small enough to give the conclusion of the lemma.

5. The foliation. Let $V$ be a local variety of codimension 1 in $C^{n}$ containing the origin. Near $0, V$ is defined by an analytic function $f(z)=f_{k}(z)+f_{k+1}(z)$ $+\cdots$ with $f_{j}(z)$ homogeneous of degree $j$ and $f_{k}(z) \not \equiv 0$. Then the tangent cone of $V$ at 0 is $C(V, 0)=\left\{z \in C^{n}: f_{k}(z)=0\right\}$, which we shall also consider as a variety in $C P^{n-1}$. If $\alpha \notin C(V, 0)$ then $f(z)$ restricted to any linear space $L \subseteq C^{n}$ containing $\alpha$ does not vanish identically because $f_{k}(z)$ does not.

Consider the set $I_{j}=\{t \in[0,1]$ : $\operatorname{Ord} f(z, t) \leqslant j\}$, which is open since $f$ is continuous. By the compactness of $[0,1]$ there exists an $N<\infty$ such that $I_{N}=[0,1]$. For $t \in \operatorname{int}\left(I_{j} \backslash I_{j-1}\right), \operatorname{Ord} f(z, t)$ is constant in a neighborhood of $t$. Furthermore $\bigcup_{j=1}^{N} \operatorname{int}\left(I_{j} \backslash I_{j-1}\right)$ is dense in $[0,1]$.

LemMa 6. Assume that $0 \in E$, and set $C(S, 0)=\cup_{t \in[0,1]} C(V(t), 0)$. Then $C(S, 0)$ has zero measure and is first Baire category in $C P^{n-1}$.

Proof. Since $I_{j}$ is open it is a countable union of open intervals, and let $I_{j k}=(a, b)$ be one such interval. If each set $C_{j k}=\cup_{t \in I_{j k} \backslash I_{j-1}} C(V(t), 0)$ has zero measure and is first category, then the same holds for $C(S, 0)=\cup_{j, k} C_{j k}$.

Consider the map $t \rightarrow f_{j}(z, t)$ for $t \in I_{j} \backslash I_{j-1}$. If $t \in I_{j} \cap \partial\left(I_{j-1}\right)$, then $f_{j}(z, t) \neq 0$. Therefore by condition (ii) on $f$, for each $\varepsilon>0$, the set

$$
\tilde{C}_{j k}(\varepsilon)=\left\{(z, t) \in C^{n} \times\left((a+\varepsilon, b-\varepsilon) \backslash I_{j-1}\right): f(z, t)=0\right\}
$$

is locally a $C^{1}$-manifold, has finite $(2 n-1)$-dimensional measure and is nowhere dense in $C^{n} \times R$.

If $\pi: C^{n} \times R \rightarrow C P^{n-1}$ denotes the standard projection $\pi(z, t)=[z]$, then $C_{j k}=\cup_{\varepsilon>0} \pi \tilde{C}_{j k}(\varepsilon)$ has measure zero and is first category in $C P^{n-1}$.

Lemma 7. Let $f(z, \zeta)$ be defined by (4.4), and suppose $f \in \mathcal{C}^{2}$. Then there exists $\delta>0$ such that $0 \notin F\left(\zeta_{1}, \zeta_{2}\right)(\partial B(0,1))$ if $\zeta_{1} \neq \zeta_{2}$ and $\left|\operatorname{Im} \zeta_{j}\right|<\delta, j=1,2$.

PRoof. Let us show first that there exists $\delta_{1}>0$ such that if $\left|\zeta_{1}-\zeta_{2}\right|<\delta_{1}$ and $\left|\operatorname{Im} \zeta_{j}\right|<\delta, j=1,2$, then $0 \notin F\left(\zeta_{1}, \zeta_{2}\right)(\partial B(0,1))$. By (ii), $\{z \in \partial B(0,1)$ : 
$\left.f(z, \theta)=d_{\theta} f(z, \theta)=0\right\}=\varnothing$. Thus for some small $k^{\prime}$,

$$
\inf _{z \in \partial B(0,1)}\left(\left|f\left(z, \zeta_{1}\right)\right|+\left|d_{\theta} f\left(z, \zeta_{2}\right)\right|\right)>k^{\prime}>0
$$

if $\zeta_{1}=\zeta_{2}=\theta$. Since this holds for each $\theta \in I$, and $I$ is compact, there exist $k$ and $\delta^{\prime}>0$ such that

$$
\inf _{z \in V\left(\zeta_{2}\right) \cap \partial B(0,1)}\left|d_{\theta} f\left(z, \zeta_{1}\right)\right|>k>0
$$

holds for $\left|\zeta_{1}-\zeta_{2}\right|<\delta^{\prime}$ and $\left|\operatorname{Im} \zeta_{j}\right|<\delta^{\prime}, j=1,2$.

Suppose that $z \in V\left(\zeta_{1}\right) \cap \partial B(0,1)$. Then

$$
\left|f\left(z, \zeta_{2}\right)\right|=\left|f\left(z, \theta_{2}\right)+i \eta_{2} d_{\theta} f\left(z, \theta_{2}\right)\right|
$$

$$
\begin{aligned}
& =\left|f\left(z, \theta_{2}\right)-f\left(z, \theta_{1}\right)+i\left(\eta_{2} d_{\theta} f\left(z, \theta_{2}\right)-\eta_{1} d_{\theta} f\left(z, \theta_{1}\right)\right)\right| \\
& =\left|\left(\left(\theta-\theta_{2}\right)+i\left(\eta_{2}-\eta_{1}\right)\right) d_{\theta} f\left(z, \theta_{2}\right)+R\left(z, \theta_{1}, \theta_{2}\right)+\eta_{1} Q\left(z \theta_{1}, \theta_{2}\right)\right|
\end{aligned}
$$

where

$$
Q\left(z, \theta_{1}, \theta_{2}\right)=d_{\theta} f\left(z, \theta_{2}\right)-d_{\theta} f\left(z, \theta_{1}\right)
$$

and

$$
R\left(z, \theta_{1}, \theta_{2}\right)=-f\left(z, \theta_{2}\right)+f\left(z, \theta_{1}\right)-\left(\theta_{1}-\theta_{2}\right) d_{\theta} f\left(z, \theta_{2}\right) .
$$

Since $f$ is $\mathcal{C}^{2}$, there exists $K$ so that $\left|Q\left(z, \theta_{1}, \theta_{2}\right)\right|<K\left|\theta_{2}-\theta_{1}\right|$. Now select $\delta_{1}>0$ sufficiently small that

$$
\delta_{1} \leqslant k(4 K)^{-1}
$$

and

$$
\left|R\left(z, \theta_{1}, \theta_{2}\right)\right| \leqslant(k / 4)\left|\theta_{2}-\theta_{1}\right|
$$

if $\left|\theta_{2}-\theta_{1}\right| \leqslant \delta_{1}$. With this choice of $\delta_{1}>0$, (5.3) yields

$$
\begin{aligned}
\left|f\left(z, \zeta_{2}\right)\right| & \geqslant\left|\eta_{2}-\eta_{1}\right| k+\left|\theta_{1}-\theta_{2}\right| k-\frac{k}{4}\left|\theta_{2}-\theta_{1}\right|-\left|\eta_{1}\right| K\left|\theta_{2}-\theta_{1}\right| \\
& \geqslant\left|\eta_{2}-\eta_{1}\right| k+\left|\theta_{1}-\theta_{2}\right| \frac{k}{2} \geqslant \frac{k}{4}\left|\zeta_{1}-\zeta_{2}\right| .
\end{aligned}
$$

Thus it follows that $V\left(\zeta_{1}\right) \cap V\left(\zeta_{2}\right) \cap \partial B(0,1)=\varnothing$, or $0 \notin F\left(\zeta_{1}, \zeta_{2}\right)(\partial B(0$, 1)).

Now consider the set

$$
T\left(\delta_{1}\right)=\left\{\left(\theta_{1}, \theta_{2}\right) \in I \times I:\left|\theta_{1}-\theta_{2}\right| \geqslant \frac{1}{2} \delta_{1}\right\} .
$$

For $\left(\theta_{1}, \theta_{2}\right) \in T\left(\delta_{1}\right), 0 \notin F\left(\theta_{1}, \theta_{2}\right)(\partial B(0,1))$ since $V\left(\theta_{1}\right) \cap V\left(\theta_{2}\right)=\varnothing$. Thus 
there exists $\delta\left(\theta_{1}, \theta_{2}\right)$ such that $0 \notin F\left(\zeta_{1}, \zeta_{2}\right)(\partial B(0,1))$ if $\left|\zeta_{j}-\theta_{j}\right|<\delta\left(\theta_{1}, \theta_{2}\right), j$ $=1,2$. Since $T\left(\delta_{1}\right)$ is compact, there exists $\delta_{2}$ such that if $\operatorname{dist}\left(\left(\zeta_{1}, \zeta_{2}\right), T\left(\delta_{1}\right)\right)$ $<\delta_{2}$, then $0 \notin F\left(\zeta_{1}, \zeta_{2}\right)(\partial B(0,1))$. Thus $\delta=\min \left\{\delta_{1}, \delta_{2}\right\}$ has the desired property.

The following corollary is given at this point to show how Theorem B follows directly from Lemma 7 if the singularity $E$ is assumed to be inessential. Corollary 3 , together with the reduction argument from $C^{n}$ to $C^{2}$ given in the proof of Theorem B, is sufficient for the case where $E$ is inessential.

Corollary 3. If $S \subseteq B(0,1) \backslash E$ is a $e^{2}$-analytic hypersurface in $C^{2}$ and $E$ is an inessential singularity, then the varieties $\{V(\zeta): \zeta \in I(\delta)\}$ foliate a neighborhood of $S$ for $\delta>0$ in the conclusion of Lemma 7.

Proor. Since $\bar{V}\left(\theta_{1}\right) \cap \bar{V}\left(\theta_{2}\right)=\varnothing$, it follows that $\operatorname{deg}\left(F\left(\zeta_{1}, \zeta_{2}\right), B(0,1), 0\right)$ $=0$ for $\zeta_{1} \neq \zeta_{2}, \zeta_{1}, \zeta_{2} \in I$. Since by Lemma 7 , the degree is defined and constant for $\zeta_{1} \neq \zeta_{2}, \zeta_{1}, \zeta_{2} \in I(\delta)$, it follows from (4.2) that $\bar{V}\left(\zeta_{1}\right) \cap \bar{V}\left(\zeta_{2}\right)$ $=\varnothing$.

Lemma 8. Let $E$ be a finite subset of $B(0,1) \subset C^{2}$. For the $\delta>0$ in the conclusion of Lemma 7, the varieties $\{V(\zeta): \zeta \in I(\delta)\}$ of $(4.5)$ define a foliation of a neighborhood of $S$.

Proof. What must be shown is that $V\left(\zeta_{1}\right) \cap V\left(\zeta_{2}\right)=\varnothing$, or equivalently, that $\bar{V}\left(\zeta_{1}\right) \cap \bar{V}\left(\zeta_{2}\right)=E$. By Lemma $7, \operatorname{deg}\left(F\left(\zeta_{1}, \zeta_{2}\right), B(0,1), p\right)$ is defined and constant for $\left|\operatorname{Im} \zeta_{j}\right| \leqslant \delta, j=1,2$, and for all $p \in E$. Since $V\left(\theta_{1}\right) \cap V\left(\theta_{2}\right)$ $=\varnothing$ for $\theta_{1} \neq \theta_{2}$, it follows that

$$
\sum_{p \in E} \operatorname{deg}\left(F\left(\zeta_{1}, \zeta_{2}\right), B(0,1), p\right)=\sum_{p \in E} \lim _{\varepsilon \rightarrow 0} \operatorname{deg}\left(F\left(\zeta_{1}, \zeta_{2}\right), B(p, \varepsilon), p\right)
$$

for $\zeta_{1}=\theta_{1}, \zeta_{2}=\theta_{2}$. Observe that (5.7) is equivalent to the condition $V\left(\zeta_{1}\right)$ $\cap V\left(\zeta_{2}\right)=\varnothing$. If $p \in E$ it will suffice to show that

$$
D\left(\left(\zeta_{1}, \zeta_{2}\right), p\right)=\lim _{\varepsilon \rightarrow 0} \operatorname{deg}\left(F\left(\zeta_{1}, \zeta_{2}\right), B(p, \varepsilon), p\right)
$$

is constant for $\zeta_{1}, \zeta_{2} \in I(\delta)$. Let us take $p=0$.

For fixed $\theta_{2}$, there exists, by Lemma $5, \delta\left(\theta_{2}\right)>0$ such that $D\left(\zeta_{1}, \theta_{2}\right)$ is constant for $\zeta_{1} \in I\left(\delta\left(\theta_{2}\right)\right)$. We claim that in fact we may take $\delta\left(\theta_{2}\right)=\delta$ in the conclusion of Lemma 7. Suppose $\delta\left(\theta_{2}\right)<\delta$. Let $\left\{p_{1}\left(\zeta_{1}\right), \ldots, p_{k}\left(\zeta_{1}\right)\right\}=\bar{V}_{1}\left(\zeta_{1}\right)$ $\cap \bar{V}\left(\theta_{2}\right)$. By Lemma 2 , the points $p_{1}, \ldots, p_{k}$ move continuously in $\zeta_{1}$ if $\zeta_{1} \in I(\delta)$ and if they do not bifurcate or coalesce. For $\zeta_{1} \in I\left(\delta\left(\theta_{2}\right)\right)$, the points $\left\{p_{1}\left(\zeta_{1}\right), \ldots, p_{t}\left(\zeta_{1}\right)\right\}=E$ are fixed. Thus if $\varepsilon>0$ is chosen less than $\min _{j \neq k}\left|p_{j}\left(\zeta_{1}\right)-p_{k}\left(\zeta_{1}\right)\right|$, then $0 \notin F\left(\zeta_{1}, \theta_{2}\right)(\partial B(0, \varepsilon))$. Thus $D\left(\zeta_{1}, \theta_{2}\right)$ is constant for $\left|\operatorname{Im} \zeta_{1}\right| \leqslant \delta\left(\theta_{2}\right)$, and we may take $\delta\left(\theta_{2}\right)=\delta$.

This shows that for $\zeta_{2} \in I(\delta), D\left(\theta_{1}, \zeta_{2}, 0\right)$ is constant in $\theta_{1} \neq \zeta_{2}$. By Lemma 
5 , there exists $\delta\left(\theta_{1}\right)$ such that $D$ is constant on $I\left(\delta\left(\theta_{1}\right)\right) \times\left\{\zeta_{2}\right\}$. By the argument above, it follows that $D$ is constant for $\left(\zeta_{1}, \zeta_{2}\right) \in I(\delta) \times\left\{\zeta_{2}\right\}$ and $\zeta_{1} \neq \zeta_{2}$. Thus $D$ is constant on $I(\delta) \times I(\delta) \backslash\left\{\zeta_{1}=\zeta_{2}\right\}$ which completes the proof.

ReMARK. The proof of Lemma 8 shows that for any $\varepsilon^{\prime}<\varepsilon$, there exists $\delta\left(\varepsilon^{\prime}\right)>0$ such that the varieties $\left\{V(\zeta), \zeta \in I\left(\delta\left(\varepsilon^{\prime}\right)\right)\right\}$ defined in $B\left(0,1+\varepsilon^{\prime}\right)$ give a foliation of a neighborhood $S$ in $B\left(0,1+\varepsilon^{\prime}\right)$.

Proof of Theorem B. Lemma 8 establishes Theorem B in the case $n=2$. We show how the theorem may be deduced from this special case. Let a point $p \in \overline{B(0,1)}$ be given, which by an element of $\operatorname{Aut}(B(0,1+\varepsilon))$ may be taken to be 0 . Make a unitary change of coordinates $\left(z_{1}, \ldots, z_{n}\right)$ such that

(a) $\left\{z_{2}=\cdots=z_{n}=0\right\} \in C P^{n-1} \backslash C(S)$,

(b) $E \cap\left\{z_{3}=\cdots=z_{n}=0\right\}$ is discrete.

To do this, let $T$ be any 2-plane such that $E \cap T$ is discrete. By Lemma 2, the set of 2-planes $T$ for which (b) holds is open. After a small perturbation, $T$ may be taken to contain a line satisfying (a), since by Lemma 6 , these lines are residual.

By (iii), there exists $\varepsilon^{\prime}>0$ such that $f\left(z_{1}, z_{2}, \alpha_{3}, \ldots, \alpha_{n}, \theta\right)$ is analytic for $\left|z_{1}\right|^{2}+\left|z_{2}\right|^{2}<1,\left|\alpha_{3}\right|^{2}+\cdots+\left|\alpha_{n}\right|^{2}<\left(\varepsilon^{\prime}\right)^{2}$, and $\theta \in I$. Since $\left\{z_{2}=\cdots\right.$ $\left.=z_{n}=0\right\} \notin C(S)$ it follows that $f\left(z_{1}, 0, \ldots, 0, \theta\right) \not \equiv$ for all $\theta \in I$. Thus we have an analytic hypersurface

$$
S \cap\left\{z_{3}=\alpha_{3}, \ldots, z_{n}=\alpha_{n}\right\}=S(\alpha) \subseteq B(0,1) \backslash E(\alpha)
$$

where $E(\alpha)=E \cap\left\{z_{3}=\alpha_{3}, \ldots, z_{n}=\alpha_{n}\right\}$ and $B(0,1) \subseteq C^{2}$.

Now it must be shown that there exists $\delta(p)>0$ such that if $|\alpha|<\delta(p)$, then the varieties $\{V(\zeta, \alpha): \zeta \in I(\delta(p))\}$ define a foliation in a neighborhood of $S(\alpha)$. By Lemma 8, it will suffice to show that the $\delta(\alpha)$ of Lemma 7 may be bounded below independent of $\alpha$ if $|\alpha|<\varepsilon^{\prime}$ is small enough. Observe that $\delta_{1}$ in the proof of Lemma 7 was subject only to the restrictions (5.2), (5.4), and (5.5). If $\varepsilon^{\prime}>0$ is chosen small enough, $\delta_{1}>0$ may be chosen to satisfy (5.2), (5.4), and (5.5) for $f(z, \alpha)$ if $|\alpha| \leqslant \varepsilon^{\prime}$. Since $\left\{|\alpha| \leqslant \varepsilon^{\prime}\right\} \times T\left(\delta_{1}\right)$ is compact, with $T\left(\delta_{1}\right)$ as in (5.6), it follows that $\delta_{2}$ may be chosen so that $0 \notin F\left(\zeta_{1}, \zeta_{2}, \alpha\right)$ - $(\partial B(0,1))$ if $|\alpha|<\delta_{2}$ and $\operatorname{dist}\left(\left(\zeta_{1}, \zeta_{2}\right), T\left(\delta_{1}\right)\right)<\delta_{2}$. Thus if $\delta=\min \left\{\delta_{1}, \delta_{2}\right.$, $\left.\varepsilon^{\prime}\right\}$, the conclusion of Lemma 7 holds for $\left(f\left(z, \alpha, \zeta_{1}\right), f\left(z, \alpha, \zeta_{2}\right)\right)$ with $|\alpha| \leqslant \delta$.

From this it follows that for $|\alpha| \leqslant \delta, \bar{V}\left(\zeta_{1}, \alpha\right) \cap \bar{V}\left(\zeta_{2}, \alpha\right)=E(\alpha)$ for $\left(\zeta_{1}, \zeta_{2}\right)$ $\in I(\delta) \times I(\delta), \zeta_{1} \neq \zeta_{2}$. Thus $\bar{V}\left(\zeta_{1}\right) \cap \bar{V}\left(\zeta_{2}\right)=E \cap B(0, \delta)$. Since there exists $\delta(p)>0$ for each $p \in \overline{B(0,1)}$ and $B(0,1)$ is compact, it follows that $\delta>0$ may be chosen sufficiently small that $\bar{V}\left(\zeta_{1}\right) \cap \bar{V}\left(\zeta_{2}\right)=E$ for $\left(\zeta_{1}, \zeta_{2}\right) \in I(\delta)$ $\times I(\delta), \zeta_{1} \neq \zeta_{2}$. This shows that the varieties $\{V(\zeta)\}$ are indeed a foliation.

PROOF OF THEOREM $B^{\prime}$. If $f(z, \theta)$ is real analytic, there exists $\delta>0$ such that $f_{0}(z, \zeta)$ is analytic on $B(0,1+\varepsilon) \times I(\delta)$, where $f_{0}$ is the analytic continuation of $f$. Note that in general $f_{0}(z, \zeta)$ is different from the function $f(z, \zeta)$ defined 
in (4.4), and thus the varieties

$$
V^{\prime}(\zeta)=\left\{z \in B(0,1) \backslash E: f_{0}(z, \zeta)=0\right\}
$$

will differ from those defined in (4.5). By (4.4), we see that

$$
\left|f(z, \theta+i \eta)-f_{0}(z, \theta+i \eta)\right| \leqslant K_{0}|\eta|^{2}
$$

for $z \in B(0,1)$ and $\theta \in I$. The proofs of Lemmas 5,7 and 8 can be modified to accommodate the new function $f_{0}(z, \zeta)$, and the proof of Theorem B, applied to the function $f_{0}(z, \zeta)$, yields Theorem $\mathrm{B}^{\prime}$.

6. Proof of Theorem D. We use the foliation of the preceding section to construct a function $F(z)$ with the property that

(a) $F$ is meromorphic on $B(0,1)$ and the pole set of $F$ is $V\left(\zeta_{0}\right) \subseteq \Omega^{-}$,

(b) $\left.F\right|_{\Omega^{+}} \in H^{\infty}\left(\Omega^{+}\right)$.

Observe that for any real $\theta$, the function $f_{j}(z)=(f(z, \theta))^{j+1} F(z)$ will be an element of $A^{j}\left(\Omega^{+}\right)$. Thus the existence of $F(z)$ shows that $\Omega^{+}$is a domain of holomorphy of type $A^{j}$ for all $j<\infty$.

For any real $\theta_{0}$, and any $\zeta_{0}$ such that $V\left(\zeta_{0}\right) \subset \Omega^{-}$, set $F(z)=f\left(z, \theta_{0}\right)$ $\cdot\left(f\left(z, \zeta_{0}\right)\right)^{-1}$. We shall show that $F$ satisfies (b).

Recall that $F$ is defined on $B(0,1+\varepsilon)$, and let $\bar{V}(\zeta), V(\zeta), S$ and $E$ represent their natural extensions to $B(0,1+\varepsilon)$. We claim that it suffices to show that $F$ is bounded on $S \cap B(0,1)$. Note that $F$ is obviously bounded on $\Omega^{+} \cap \partial B(0,1)$, and thus this claim is a consequence of the following lemma.

If $G$ is meromorphic in a domain $\Omega$, let $\alpha \in C$ be called a boundary limit of $G$ at $p \in \partial \Omega$ if there exists a sequence $\left\{z_{j}\right\} \subseteq \Omega$ such that $\lim _{j \rightarrow \infty} z_{j}=p$ and $\lim _{j \rightarrow \infty} G\left(z_{j}\right)=\alpha$.

LEMMA 9. Let $\Omega$ be a bounded open set in $C^{n}$ with $n \geqslant 2$, and let $G$ be meromorphic on $\Omega$. Let $E$ be a local variety of codimension 2 in $\Omega$. If $G$ is the set of indeterminacy of $G$, then $G(\Omega \backslash g) \subseteq\{\alpha: \alpha$ is a boundary limit of $G\}$. In particular, taking the moduli of the elements in these two sets, we obtain

$$
\sup _{z_{0} \in \partial \Omega \backslash E} \limsup _{z_{j} \rightarrow z_{0}}\left|G\left(z_{j}\right)\right|=\sup _{z \in \Omega}|G(z)| \text {. }
$$

Proof. For fixed $z_{0} \in \Omega \backslash 9$, set $W=\left\{z\right.$ : for some $\left.z_{j} \rightarrow z, G\left(z_{j}\right) \rightarrow G\left(z_{0}\right)\right\}$. If $G\left(z_{0}\right)$ is not a boundary limit at any point of $\partial \Omega \backslash E$, then it follows that $\bar{W} \cap(\partial \Omega \backslash E)=\varnothing$, where $\bar{W}$ denotes the closure of $W$ in $C^{n}$. From this, we see that $\bar{W} \backslash E$ is closed in $C^{n} \backslash E$. Furthermore, $\bar{W} \cap \Omega$ is a variety in $\Omega$, so it follows that $\bar{W}$ is a variety in $C^{n}$, since $E$ is a removable singularity for a variety of codimension 1 . On the other hand, since $W$ is bounded, it is compact and must be a finite set. Thus $W$ can have codimension 1 only if 
$n=1$, which is contrary to assumption.

To show that $F$ is bounded on $S$, we first show that the restriction $\left.F\right|_{V(\theta) \backslash E}=F(\theta) \in \theta(V(\theta) \backslash E)$ extends to an element of $\theta(V(\theta))$. Since $F(\theta)$ is meromorphic on $V(\theta)$, the set $E$ must be a pole set for $F(\theta)$ if $F(\theta)$ is not analytic on $V(\theta)$.

We show that $F(\theta)$ cannot have a pole at $E$ as follows. Pick coordinates $z=\left(z_{1}, \ldots, z_{n}\right)$ such that $E \cap\left\{z \in B(0,1+\varepsilon): z_{3}=\cdots=z_{n}=0\right\}$ is a discrete set and $0 \in E$. As before let $V(\theta) \cap\left\{z_{3}=\cdots=z_{n}=0\right\}$ $=\cup_{j=1}^{k} V_{j}$, where $V_{j}$ is irreducible at 0 and let $\left(\phi_{j}(\tau), \psi_{j}(\tau)\right)$ be a normalization near 0 . Then by Lemma 8, the order of $f_{j}(\tau, \zeta)=f\left(\phi_{j}(\tau), \psi_{j}(\tau), \zeta\right)$ in $\tau$ is constant for $|\operatorname{Im} \zeta|<\delta, \zeta \neq \theta$. Thus, on the irreducible component $V_{j}$, $F_{j}(\theta)(\tau)=f_{j}\left(\tau, \theta_{0}\right)\left(f_{j}\left(\tau, \zeta_{0}\right)\right)^{-1}$ is analytic at the origin. If $F(\theta)$ had a pole at $E$, it would follow that $\lim _{\tau \rightarrow 0} F_{j}(\theta)(\tau)=\infty$.

Define for small $\varepsilon^{\prime}>0$ the set

$$
T\left(\varepsilon^{\prime}\right)=\left\{z \in B\left(0,1+\varepsilon-\varepsilon^{\prime}\right) \cap S: \operatorname{dist}(E, z)>\varepsilon^{\prime}\right\} .
$$

It is clear that $F$ is bounded on $T\left(\varepsilon^{\prime}\right)$. To conclude the proof that $F$ $\in H^{\infty}\left(\Omega^{+}\right)$, we shall show that for $\varepsilon^{\prime}>0$ sufficiently small

$$
\sup _{z \in T\left(e^{\prime}\right)}|F(z)| \geqslant \sup _{z \in S \cap B(0,1)}|F(z)| \text {. }
$$

Let us make use of the fact that for each $p \in E$ and arbitrary $\eta>0$, there exist $\delta>0$ and a translation and rotation of coordinates $z=\left(z_{1}, \ldots, z_{n}\right)$ such that $p=0$ and $E \cap\left(\Delta^{n-2}(\delta) \times A^{2}(\eta)\right)=\varnothing$, where we write $\Delta^{n-j}(\delta)$ $=\left\{\left|z_{1}\right|^{2}+\cdots+\left|z_{n-j}\right|^{2}<\delta\right\}$ and $A^{j}(\eta)=\left\{\eta / 2<\left|z_{n-j+1}\right|^{2}+\cdots+\left|z_{n}\right|^{2}\right.$ $<\eta\}$. See, for instance, [9, p. 80]. For $p \in B(0,1+\varepsilon / 2) \cap E$, one may choose $\eta<\frac{1}{4} \operatorname{dist}(p, \partial B(0,1+\varepsilon))$ and obtain a covering of $B(0,1+\varepsilon / 2) \cap E$ by polydisks $\Delta^{n-2}(\delta) \times \Delta^{2}(\eta)$. Selecting a finite subcover, we may choose $\varepsilon^{\prime}>0$ such that $T\left(\varepsilon^{\prime}\right)>0$ contains each of the sets $\Delta^{n-2} \times A^{2}$. Thus inequality (*) follows from a maximum principle for holomorphic functions on varieties.

LEMMA 10. Let $V$ be $a$ variety of codimension 1 in $\Delta^{n-2}(\delta) \times \Delta^{2}(\eta)$. If $f \in \mathcal{O}(V)$, then

$$
\sup _{z \in V \cap\left(\Delta^{n-2}(\delta) \times A^{2}(\eta)\right)}|f(z)|=\sup _{z \in V \cap\left(\Delta^{n-2}(\delta) \times \Delta^{2}(\eta)\right)}|f(z)| .
$$

Proof. If $\left(z_{1}, \ldots, z_{n-2}\right)$ is fixed, then this is seen to be an easy consequence of Theorem 2G in $[9$, p. 108].

\section{REFERENCES}

1. K. Diederich and J. E. Fornaess, Exhaustion functions and Stein neighborhoods for smooth pseudoconvex domains, Proc. Nat. Acad. Sci. U.S.A. 72 (1975), 3279-3280. 
2. A strange bounded smooth domain of homomorphy, Bull. Amer. Math. Soc. 82 (1976), $74-76$.

3. J. J. Kohn, Global regularity for $\bar{\partial}$ on weakly pseudo-convex manifolds, Trans. Amer. Math. Soc. 181 (1973), 273-292. MR 49 \#9442.

4. P. Lelong, Fonctionnelles analytiques et fonctions entieres ( $n$ variables), Presses Univ. Montréal, Montréal, 1968, p. 31.

5. P. H. Rabinowitz, A note on topological degree theory for holomorphic maps, Israel J. Math. 16 (1973), 46-52. MR 49 \# 10909.

6. N. Sibony, Prolongement analytiques des fonctions holomorphes bormées, C. R. Acad. Sci. Paris Sér. A 275 (1972), 973-976. MR 47 \# 7062.

7. K. Spallek, Differenzierbare und holomorphe Funktionen auf analytischen Mengen, Math. Ann. 161 (1965), 143-162. MR 33 \#4328.

8. V. S. Vladimirov, Methods of the theory of functions of many complex variables, "Nauka", Moscow, 1964; English transl., Scripta Technica, M.I.T. Press, Cambridge, Mass., 1966. MR 30 \#2163; 34 \# 1551 .

9. H. Whitney, Complex analytic varieties, Addison-Wesley, Reading, Mass., 1972.

10. C. Rea, Levi-flat submanifolds and holomorphic extensions of foliations, Ann. Scuola Norm. Sup. Pisa 26 (1972), 665-681.

Courant Institute of Mathematical Sciences, New York Universtty, New York, New YoRx 10012

Current address: Department of Mathematics, Princeton University, Princeton, New Jersey 08540 